\title{
An operational flash-flood forecasting chain applied to the test cases of the EU project HYDROPTIMET
}

\author{
A. C. Taramasso ${ }^{1,2}$, S. Gabellani ${ }^{1}$, and A. Parodi ${ }^{1}$ \\ ${ }^{1}$ CIMA, Centro di ricerca, Interuniversitario in Monitoraggio Ambientale, University of Genoa and Basilicata, Savona, Italy \\ ${ }^{2}$ DIST, Dipartimento di Informatica, Sistemistica e Telematica, University of Genoa, Genoa, Italy
}

Received: 4 April 2005 - Revised: 20 July 2005 - Accepted: 27 July 2005 - Published: 21 September 2005

Part of Special Issue "HYDROPTIMET"

\begin{abstract}
The application of a flash-flood prediction chain, developed by CIMA, to some testcases for the Tanaro river basin in the framework of the EU project HYDROPTIMET is presented here. The components of the CIMA chain are: forecast rainfall depths, a stochastic downscaling procedure and a hydrological model.

Different meteorological Limited Area Models (LAMs) provide the rainfall input to the hydrological component. The flash-flood prediction chain is run both in a deterministic and in a probabilistic configuration. The sensitivity of forecasting chain performances to different LAMs providing rainfall forecasts is discussed. The results of the application show how the probabilistic forecasting system can give, especially in the case of convective events, a valuable contribution in addressing the uncertainty at different spatio-temporal scales involved in the flash flood forecasting problem in small and medium basins with complex orography.
\end{abstract}

\section{Introduction}

The project HYDROPTIMET is a European MEDOCC project coordinated by the Piedmont Region (Italy) and CIMA is one of the Italian partners. This project was especially designed to define some general guidelines for the management of the principal sources of uncertainty arising from the application of operational flash-flood prediction chains in small watersheds with complex orography, typical of the Mediterranean area. Moreover, the development of a safe, reliable and fast system for the exchange of hydrometeorological data among the countries stretching along the north-western coastlines of Mediterranean sea was emphasized.

This paper presents the results provided by a flash-flood prediction chain, developed by CIMA, for some HYDROPTIMET testcases representative of hydrometeorological sce-

Correspondence to: A. C. Taramasso

(act@cima.unige.it) narios typical of medium and small mediterranean watersheds.

For such class of catchments, characterized by a response time of a few hours, social safety demands that hydrologists provide reliable prediction of ground effects at least $12-24 \mathrm{~h}$ in advance (Siccardi, 1996). Therefore, to accomplish this task, it is necessary to use rainfall numerical model forecasts as input to rainfall runoff models.

Two major sources of uncertainty arise, however, in the coupling of meteorological and hydrological models (Ferraris et al., 2002): the "external" uncertainty (numerical approximation, boundary and initial conditions) at the meteorological scale and the "internal" uncertainty of the hydrological processes involved.

The external uncertainty may be quantified by using EPSlike and LEPS-like approaches (Molteni et al., 2001; Montani et al., 2001, 2003). However, such aspect is not considered here, because this special topic was not addressed explicitly within the HYDROPTIMET project framework.

Impossible as it is to reach a reliable deterministic modeling of rainfall at the hydrologically demanded scale (Alderman and Drogemeir, 2002; Ferraris et al., 2002; Siccardi et al., 2005), the internal uncertainty can be addressed by using a stochastic downscaling procedure that generates ensemble rainfall predictions at small scales.

The CIMA operational chain uses meteorological model predictions of LAMI (Limited Area Model Italian) with horizontal resolution of $7.0 \mathrm{~km}$, a stochastic downscaling procedure based on a multifractal downscaling model (Deidda, 2000) and a hydrological model DRiFt (Giannoni et al., 2005). However within the present work a few LAMs were tested together with LAMI at $7.0 \mathrm{~km}$. They are LAMI at 2.8 $\mathrm{km}$ (courtesy of T. Paccagnella, ARPA-SIM), Bolam (courtesy of A. Buzzi, CNR-ISAC) and Moloch (courtesy of A. Buzzi, CNR-ISAC).

The first three sections of this paper give a short overview of the meteorological models, of the stochastic downscaling procedure and of the hydrological model used in this work. In the fourth section, two case studies which interested the 
Tanaro river basin in north western Italy (14-18 November 2002 and 24-26 November 2002) are presented: the first event had a prevailing convective nature, while the second one was stratiform.

In the fifth section the performances of the direct coupling of the LAM models with the hydrological model, without a stochastic downscaling, are evaluated. This approach leads to a significant underestimation of the flow peak and of the flow volume, especially when the meteorological conditions are likely to develop convective cells.

The sixth section contains the application of the probabilistic approach which adopts, when necessary, the stochastic downscaling model to generate small scale spatiotemporal rainfall events, and shows how this approach can quantify the probability of occurrence of a flood conditioned to the meteorological forecast. The choice of using the downscaling model is mainly conditioned by the type of synoptic conditions. In the case of a LAM forecast meteorological scenario with a prevailing convective nature, the disaggregation model plays a decisive role in generating rainfall fields at small spatio-temporal scales and solving problems of uncertainty at "internal-scale".

Conversely, when the meteorological forecast has a widespread stratiform character, the downscaling unit does not improve the quality of the hydrometeorological forecast for civil protection purposes. In order to support this statement, in this work, the hydro-meteorological chain is applied to the proposed events in two possible configurations: the first one with the downscaling part suppressed, the second one activating it. The results of this experiment are discussed in detail focusing on the sensitivity of the chain results depending on the different LAMs used as input.

Therefore, in the case of events characterized by convective activity, the major part of the underestimation of the peak flow in the analyzed hydrometric sections disappears when the meteorological quantities are disaggregated at a scale appropriate for hydrology. In the case, instead, of stratiform events, the contribution of the downscaling procedure is less relevant.

\section{Meteo-hydrologic forecasting chain}

\subsection{Meteorological models}

As said above in this work, a few LAM models are used: LAMI, Bolam and Moloch.

The LAMI model, derived from Lokal Model (DWD, Germany), is operated in Italy by the regional meteorological service ARPA-SIM: LAMI is the Italian LAM model of reference (Direttiva del Presidente del Consiglio dei Ministri, 27 February 2004, G.U. no. 59 dell'11/3/2004, suppl. ord. no. 39).

This model is formulated by using the 3D primitive hydrothermodynamical equations describing compressible nonhydrostatic flow in a moist atmosphere without any scale approximations (Doms and Schaettler, 1999). LAMI adopts a generalized terrain-following coordinate, an Arakawa $\mathrm{C} /$ Lorenz grid and a second order horizontal and vertical differencing approach. The physics of the model used in this work is based on several parameterization packages: a level 2.5 moist turbulence parameterization, a $\delta$-two stream radiation scheme (Ritter and Geleyn, 1992), a two-layer soil model (Jacobson and Heise, 1982) and a Louis formulation of surface fluxes (1979). The model includes a two-category ice scheme. In the present work the $7.0 \mathrm{~km}$ LAMI version adopts a Tiedtke (1989) parameterization of moist convection, while the $2.8 \mathrm{~km}$ LAMI configuration, which is nested in the $7.0 \mathrm{~km}$ LAMI forecasts, resolves explicitly the convection. The $7.0 \mathrm{~km}$ LAMI provides rainfall depth forecasts at a reliable temporal resolution of $3 \mathrm{~h}$, while the $2.8 \mathrm{~km}$ LAMI at temporal resolution of $1 \mathrm{~h}$.

The Bologna Limited Area Model (Bolam) is a hydrostatical limited area model which uses 3D primitive equations in $\sigma$ coordinates (Buzzi et al., 1994, 2003a; Buzzi and Foschini, 2000), developed at ISAC-CNR in Bologna.

A three-layer soil model is used to describe surface processes, while radiation is parameterized through the ECMWF scheme (Morcrette, 1991; Morcrette et al., 1998). The microphysical scheme (Schultz, 1995) adopts five prognostic variables (cloud ice, cloud water, rain, snow and graupel). Bolam provides a moist convection parameterization based on the Kain-Fritsch approach (1990).

The 3D non-hydrostatic, explicitly convection resolving model Moloch has been created recently at ISAC-CNR for very high resolution short-range weather prediction and research purposes (Buzzi et al., 2003b). Moloch is formulated in terms of a fully compressible set of equations with prognostic variables (pressure, temperature, horizontal and vertical velocity components) represented on the Arakawa C grid. The model uses terrain-following vertical coordinates which become horizontal surfaces as they move away from the earth surface. Time integration is performed with an implicit scheme for the vertical propagation of sound waves, while the remaining terms are computed with explicit, timesplitting schemes. The Moloch microphysical scheme is based on the work of Drofa (2003). Some of the other physical schemes (e.g. radiation, vertical diffusion, turbulent surface fluxes and soil models) are at the moment similar to those of Bolam. For the testcases here discussed, Moloch with horizontal resolution of $2.2 \mathrm{~km}$ is nested into BOLAM $(6.5 \mathrm{~km})$.

The Bolam and Moloch rainfall depth forecasts are available at a temporal resolution of $2 \mathrm{~h}$.

\section{$2.2 \delta \sigma$ Disaggregation model}

The downscaling model used here to fill the gap between meteorological and hydrological scales is a multifractal model ${ }^{1}$ based on a wavelet transformation (Deidda, 2000). The

\footnotetext{
${ }^{1} \mathrm{~A}$ new disaggregation model for the generation of precipitation fields at fine temporal and spatial scales, not based on the selfsimilarity and self-affinity hypothesis (Rebora et al., 2005), is currently being made operational.
} 


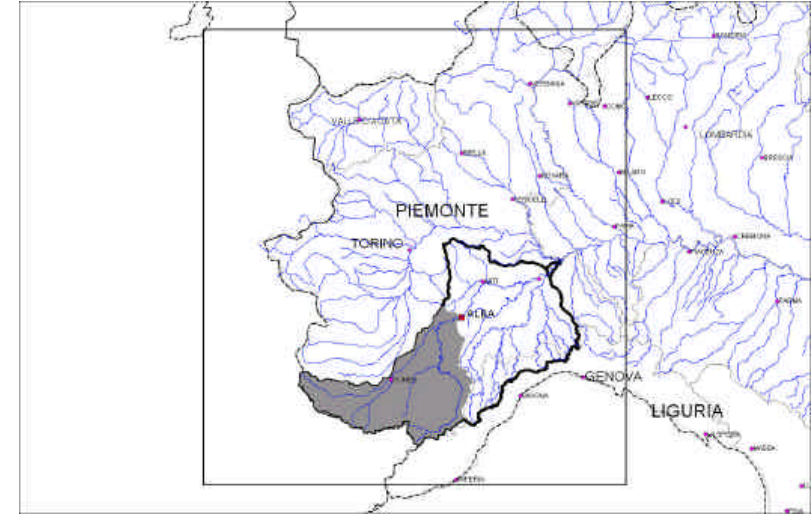

Fig. 1. Disaggregation domain (Coordinates LonSW: 6.20; LatSW: 43.80; LonNE: 9.20; LatNE: 46.20).

model adopts a positive definite wavelet expansion in $R^{n}$ and coefficients extracted from a stochastic cascade. Each term is obtained by multiplying the mother element by a random variable $h$, called generator. The choice of $h$ characterizes the multifractal behavior of the synthetic fields produced by the model. We follow Deidda (2000) and use a log-Poisson distribution governed by two parameters $b$ and $C$ calibrated in the cited paper, identical for all LAMs and without any relation to the grid size of the models. The distribution of the rainfall fields generated by the downscaling procedure is consistent with those observed in radar measurements of the rainfall rate (Deidda, 2000).

The procedure of the operational application of this downscaling model is described in Ferraris et al. (2002). For the testcases here discussed, the volume forecast by each LAM is disaggregated over a $256 \times 256 \mathrm{~km}$ domain centered on the studied area (Fig. 1). For each forecast, 100 precipitation events, equally probable and independent, with a resolution of $0.5 \times 0.5$ are generated.

\subsection{Hydrologic model}

DRiFt (DischargeRiverForecast) is a linear semi-distributed model based on a geomorphologic approach (Giannoni et al., 2000, 2005). DRiFt focuses on the main characteristics of the hydrograph: peak and time to peak. Its parameters have an intuitive and direct physical meaning and they are calibrated by forcing computed hydrographs to respect some geomorphologic hypothesis (Giannoni et al., 2005).

This hydrological model takes into account the spatial variation of inputs such as rainfall, morphologic, geological and anthropic characteristics of the basin, but it is lumped in parameters. That is why, DRiFt is in the class of calibratedparameters, semi-distributed models. This model presents most of the advantages of both distributed and lumped models and it relies on an efficient description of the drainage system: hillslopes and channel networks. These are addressed with two kinematic scales, which determine the base of the geomorphologic response of the basin. The morphologic

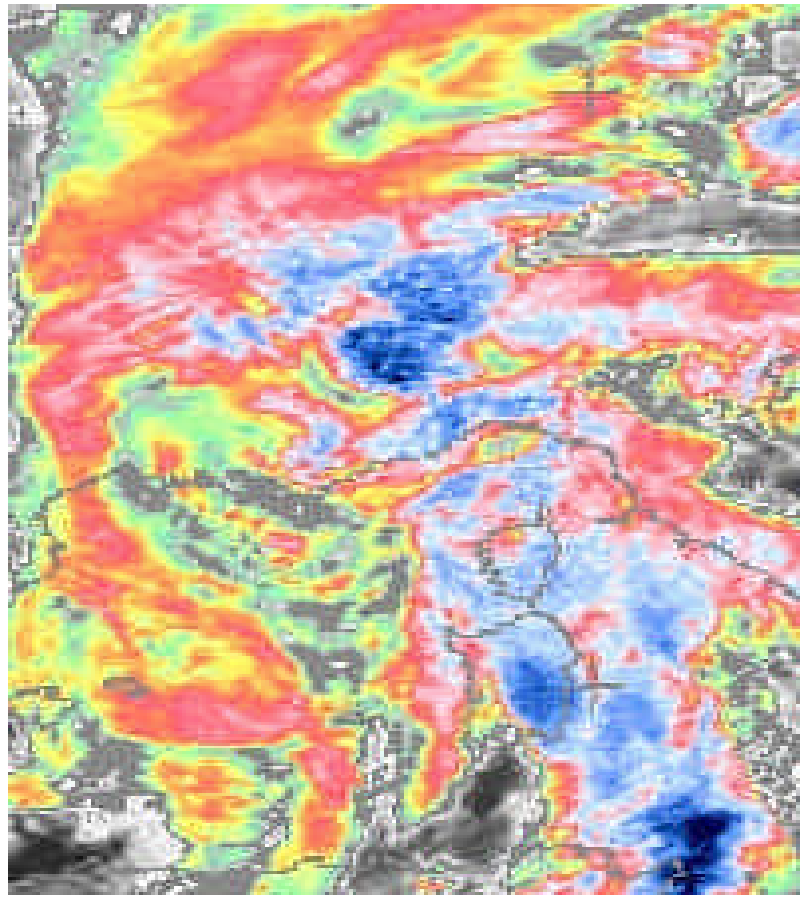

Fig. 2. IR satellite image (16 November 2002, 03:30 GMT). Areas with low values of top IR radiance temperature (blue color) are characterized by highest probability of heavy convective rainfall.

module is combined with a simple representation of soil infiltration properties (SCS-CN, 1985). Discharge at any location along the drainage network is evaluated by applying the convolution integral of the time-variant Instantaneous Unit Hydrograph.

The parameters of the model have an intuitive and direct physical meaning and they are calibrated over a number of catchments, including the target area of study (Giannoni et al., 2000). The model gives good results in geomorphologic homogeneous catchments with no variation in the parameters, regardless of the basin size and rainfall intensities. Its robustness, according to the parameters of the model, makes it not only a good research tool but also a convenient link within an operational forecasting chain.

$$
Q(t)=\int_{B} M\left(t-\frac{d_{0}(x)}{v_{0}}-\frac{d_{1}(x)}{v_{1}}, x\right) d x .
$$

\section{Case studies}

Two events, within those considered in the HYDROPTIMET project, are here investigated: 14-18 November 2002 (event 1) and 24-26 November 2002 (event 2) for the Tanaro basin closed at Alba (Fig. 1). The Tanaro basin is located in the north western part of Italy and closed at Alba has an area of $3420 \mathrm{~km}^{2}$.

The synoptical scenario for these two events was mainly characterized by a sequence of two deep troughs 


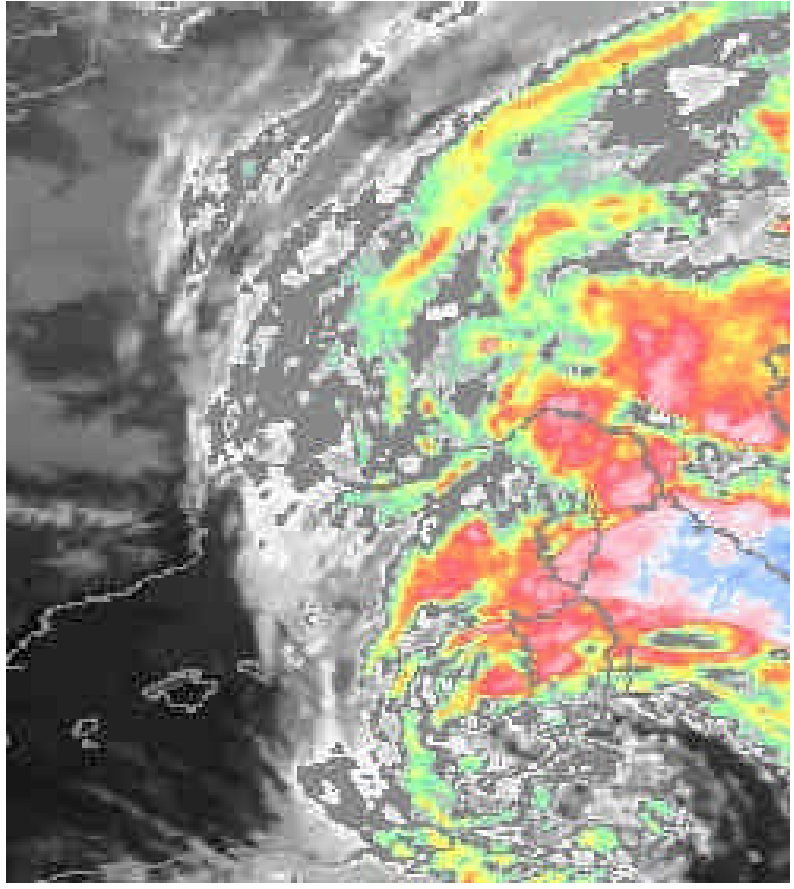

Fig. 3. IR satellite image (26 November 2002, 11:30 GMT). Areas with low values of top IR radiance temperature (blue color) are characterized by highest probability of heavy convective rainfall.

progressively extending their influence over the Mediterranean basin.

In both cases, due to a minimum isolated south of Sardinia, the flow became southerly and moist air impinged on the Alps and the Appenines, thus generating relevant precipitations in the area of northern Piedmont (Toce basin), south western Piedmont (event 1, Tanaro basin) and western Liguria.

However, as testified by the analysis of some classic stability indices (showalter index and sweat index; Mueller et al., 1993) evaluated at the sounding station of Cuneo-Levaldigi in the Tanaro river are, event 1 was characterized by convective activity, while event 2 was mainly stratiform. This result is also supported by Meteosat images (Fig. 2) which showed, during event 1 , the development of cloud structures with low values of top IR radiance temperature especially in the south western mountainous part of the Tanaro catchment. Such cloud structures are currently interpreted as areas characterized by convective activity (Bolla et al., 1996; Lanza and Conti, 1995; Velden et al., 1994). In the case of event 2 , these areas of convective activity were not observed over the Tanaro basin area (Fig. 3).

$S W I=T_{500}-T_{p 500}$.

\section{Deterministic flash-flood prediction}

First we follow a "deterministic" approach by suppressing the disaggregation step. The sequence of rainfall fields fore-

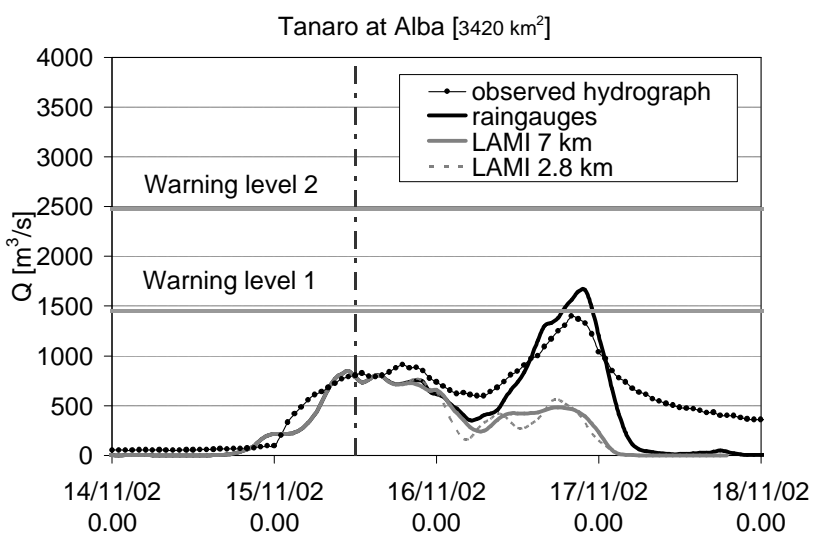

Fig. 4. Run of the hydrologic model using recorded rainfall and rainfall forecasted by the LAMI for the HYDROPTIMET test case of 14-18 November 2002. The vertical line represents the starting time of the hydrological forecast.

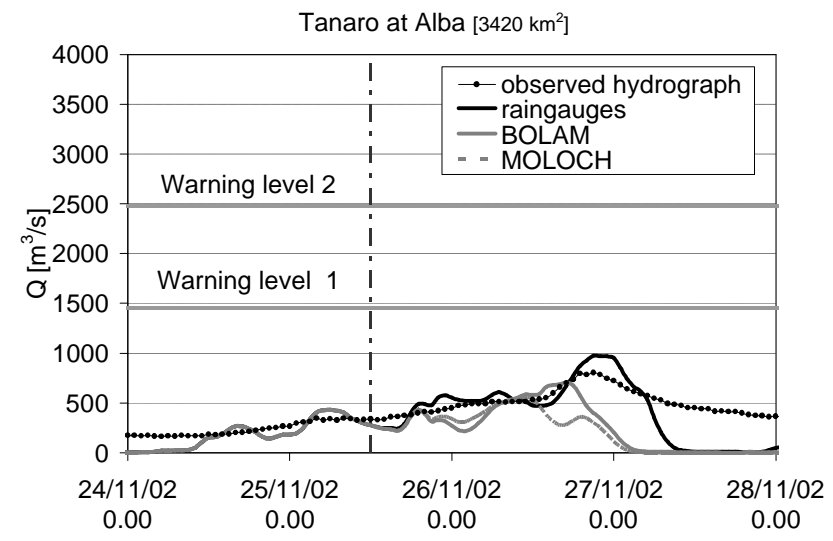

Fig. 5. Run of the hydrologic model using recorded rainfall and rainfall forecasted by the Bolam and Moloch for the HYDROPTIMET test case of 24-28 November 2002. The vertical line represents the starting time of the hydrological forecast.

cast by each LAM feeds directly the hydrological model DRiFt without the application of the disaggregation model. The hydrographs in the hydrometric station of Alba are simulated by using the observed rainfall up to 12:00 UTC 15 November, for the first event, and up to 12:00 UTC 25 November for the second event. The following $36 \mathrm{~h}$ are simulated with the rainfall fields forecast by the different LAMs. Figures 4, 5 and 6 show the results of the deterministic forecast for the two events depending on the different LAMs rainfall forecasts available: LAMI at 7 and $2.8 \mathrm{~km}$ for event 1 , all LAMs for event 2. These figures also show the simulations of the hydrographs obtained with raingauge observations and they represent the validation of the rainfall-runoff model. The results allow us to observe how the peak discharge, obtained by combining DRiFt directly with LAMs rainfall depth forecasts, underestimates the observed peaks and the discharge volumes in particular for the first event (14-18 November 2002), while in the case of event 2, mainly 


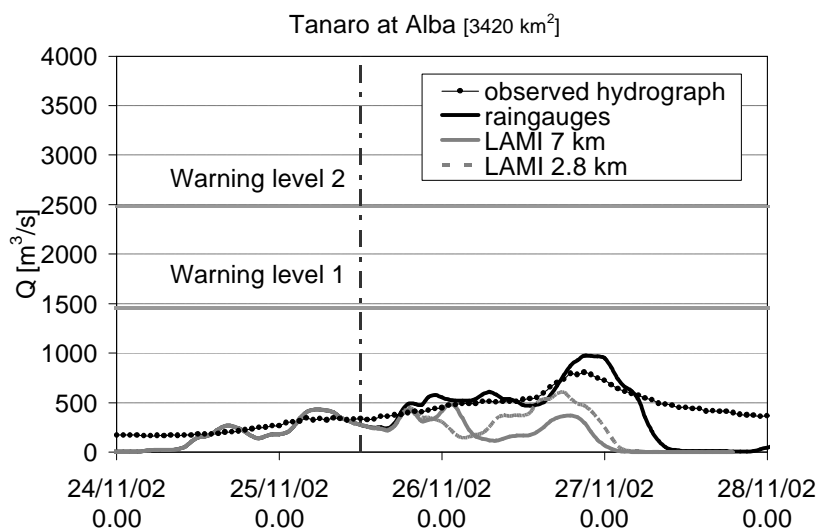

Fig. 6. Run of the hydrologic model using recorded rainfall and rainfall forecasted by the LAMI for the HYDROPTIMET test case of 24-28 November 2002. The vertical line represents the starting time of the hydrological forecast.

stratiform, there is only a slight difference between the observed and the modeled peak times.

A deeper understanding of these results can be obtained on the basis of the following considerations. All LAMs, in the case of event 1 , produce an underestimation of the observed cumulated rainfall over the Tanaro catchment (about $30 \%$ ), mainly in the mountainous part of the basin, and also a relevant uncertainty as far as the position of clusters of predicted heaviest precipitation is concerned (Fig. 7). This result is valid both for coarser and finer LAMs rainfall predictions, suggesting that for this event LAMs are unable to capture the fine scale convective activity discussed in the previous section (Fig. 3). In the case, instead, of the 24-26 November 2002 event these inconsistencies are less emphasized (Fig. 8).

The next section describes how the disaggregation step can somehow capture the small scale convective activity observed during event 1 and, therefore, compensate the major part of the underestimation of the peak flow and discharge volume arising from the direct combination of rainfall depth forecast and hydrological model.

Besides, the disaggregation is also applied to the stratiform case (event 2) in order to highlight the difference between the two cases and to show how, as expected, the contribution of the downscaling approach is less important.

\section{Probabilistic flash-flood prediction chain}

In order to account for the scale gap between meteorology and hydrology each LAM forecast is disaggregated with the multifractal model described above. The downscaling model uses as input the cumulated rainfall forecast by the LAMs and generates, for each model, 100 possible and equally likely precipitation events, independent of one another, with a spatial resolution of about $0.5 \mathrm{~km}$ and a temporal resolution
Table 1. Exceedence probability of the first warning level for the different LAMs forecasts for event 1.

\begin{tabular}{cc}
\hline \multicolumn{2}{c}{$14-16$ November 2002 event } \\
Meteorological model & Exceedence probability \\
\hline LAMI $7.0 \mathrm{~km}$ & $60 \%$ \\
LAMI $2.8 \mathrm{~km}$ & $65 \%$ \\
\hline
\end{tabular}

Table 2. Exceedence probability of the first warning level for the different LAMs forecasts for event 2.

\begin{tabular}{cc}
\hline \multicolumn{2}{c}{ 24-26 November 2002 event } \\
Meteorological Model & Exceedence Probability \\
\hline LAMI 7 km & $25 \%$ \\
LAMI 2.8 km & $23 \%$ \\
Bolam & $30 \%$ \\
Moloch & $40 \%$ \\
\hline
\end{tabular}

of about $30 \mathrm{~min}$. Then, these rainfall fields feed the rainfallrunoff model DriFt.

Each peak discharge value of the simulations has a probability of occurrence equal to: $Q_{i}^{=} \frac{i}{n+1}$, where $i=1, \ldots, n$ number of realization; $n=100$.

The results are summarized as a probability curve of exceedence of the peak discharge, conditioned to the precipitation volume in the target area derived from each model outputs.

In a civil protection framework this probabilistic curve is a very important element: an early flood warning can be issued as long as the forecast peak discharge exceeds a predetermined threshold (first warning level) in the hydrometric section of reference.

Figures 9, 10 and 11 show the cumulative distribution functions of the peaks for the Tanaro at Alba plotted on a Gumbel chart. The dotted vertical line represents the observed peak flow.

The probability of exceedence of the first warning level for the different LAMs forecasts is shown in Tables 1 and 2.

For both testcases, on the basis of the information derived simply from deterministic LAMs runs, no early warning would have been issued because no peak flow exceeded the threshold discharges in the Alba hydrometric section.

Conversely, if a probabilistic approach, based on the disaggregation step, had been considered, the civil protection scenario would have been different. In fact, in the case of event 1 , the one more intense and convective from a hydrometerological standpoint, the probability of exceedence of the threshold peak flow is more than 0.6 for LAMI $(7.0 \mathrm{~km}$ and $2.8 \mathrm{~km}$ ), thus calling for precautionary measures. Such result shows how important and useful the downscaling procedure can be in order to address the "internal-scale" uncertainty 

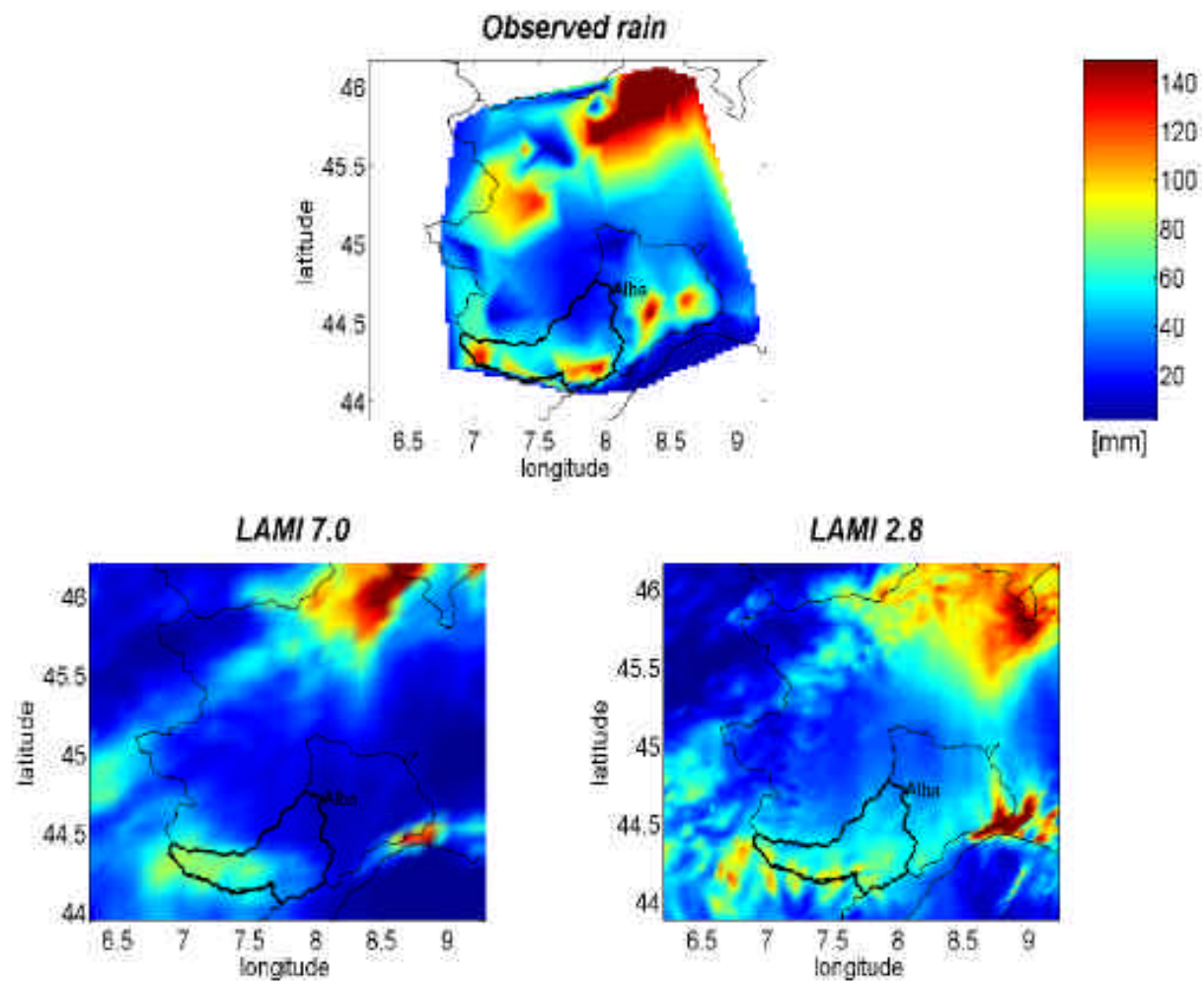

Fig. 7. Observed cumulated rainfall and forecasted cumulated rainfall for the HYDROPTIMET test case of 14-18 November 2002.

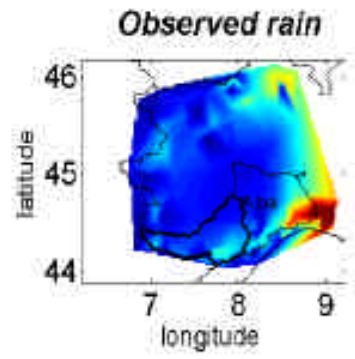

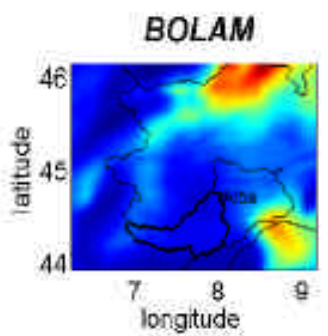

LAMI 7.0

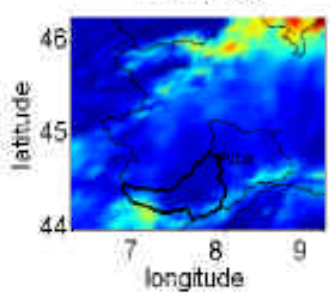

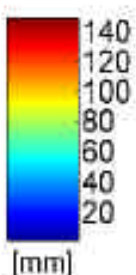

MOLOCH

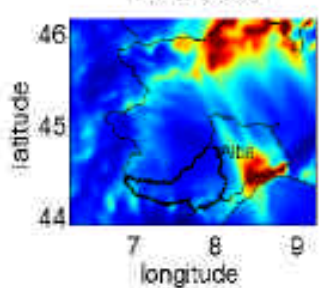

LAMI 2.8

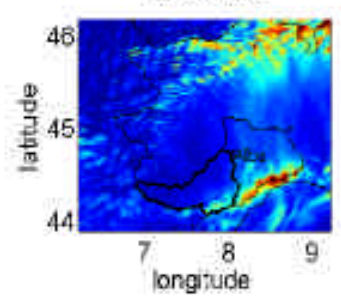

Fig. 8. Observed cumulated rainfall and forecasted cumulated rainfall for the HYDROPTIMET test case of 24-28 November 2002. 


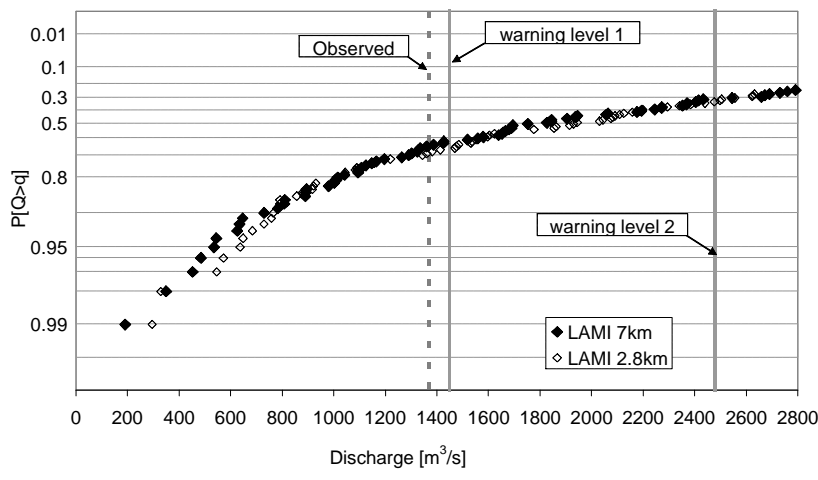

Fig. 9. Peak discharge exceedence probability plotted on a Gumbel chart for the HYDROPTIMET test case of 14-18 November 2002. The vertical dotted line represents the observed peak.

problems and to compensate the errors in the modeling of peak flow and volume discharge.

The situation would have been different for event 2 , since the probability of exceedence of the threshold peak flow would have been lower than $30-40 \%$ for all LAMs. Such aspects assess the low degree of risk associated with this event and highlights that, in the case of stratiform events, the disaggregation step is less important.

These results confirm that approaching the flash flood forecasting problem within a deterministic frame, at least for small and medium-size catchments with complex orography and for convective meteorological scenarios, cannot be considered particularly useful and reliable (Ferraris et al., 2002; Siccardi et al., 2005). It is, therefore, necessary to use a probabilistic forecasting system able to address the uncertainty at the different scales involved.

\section{Conclusions}

This paper presents the application of a flash-flood prediction chain developed at CIMA to two HYDROPTIMET testcases which interested the Tanaro river closed at Alba. Such chain in the operational configuration relies on the use of LAMI forecast rainfall depths, a stochastic downscaling procedure and a hydrological model. In the examined testcases, other different LAMs feed the hydrometeorological chain.

The results confirm that the use of a probabilistic system (Ferraris et al., 2002; Siccardi et al., 2005), able to address the uncertainty between meteorological and hydrological scales, is crucial to approach flash flood forecasting in small and medium basins with complex orography within a framework of civil protection. This is especially true in the case of event 1 (14-18 November 2002), where the disaggregation step proves able to capture the observed small scale convective activity, missed in the LAMs forecast, and, therefore, to compensate the major part of the underestimation of the peak flow and discharge volume arising from the direct combination of rainfall depth forecast and hydrological model. While in the case of event 2 (24-26 November 2002),

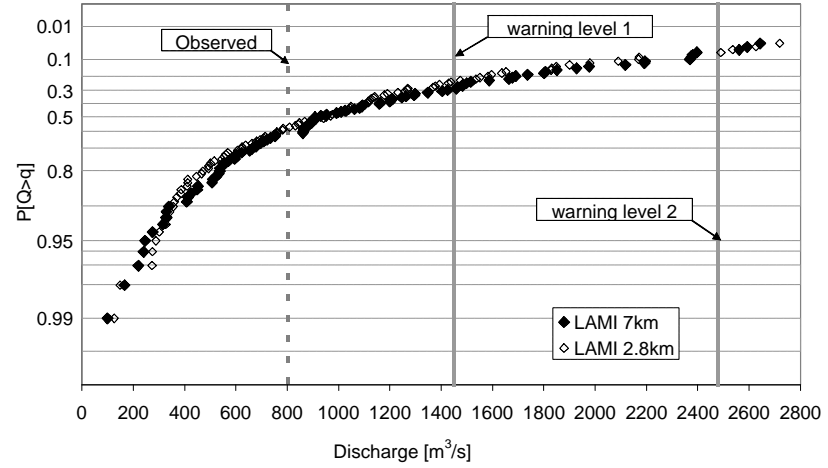

Fig. 10. Peak discharge exceedence probability plotted on a Gumbel chart for the HYDROPTIMET test case of 24-26 November 2002. The vertical dotted line represents the observed peak.

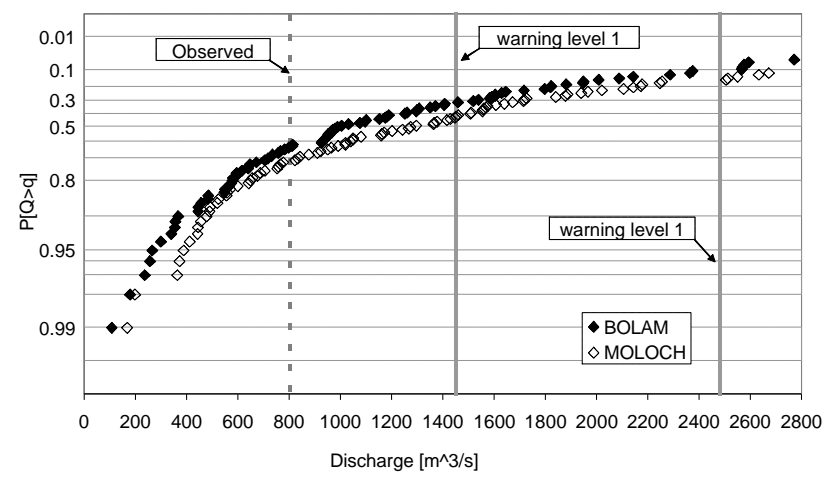

Fig. 11. Peak discharge exceedence probability plotted on a Gumbel chart for the HYDROPTIMET test case of 24-26 November 2002. The vertical line represents the The vertical dotted line represents the observed peak.

which had a prevailing stratiform nature, the contribute of the disaggregation step is less significant.

Acknowledgements. We are grateful to F. Siccardi, L. Ferraris and N. Rebora for enlighting discussions and useful comments. We are also grateful to ARPA-Piemonte, ARPA-SIM and CNR-ISAC for providing essential data.

Edited by: M.-C. Llasat

Reviewed by: L. Garrote and two other referees

\section{References}

Adlerman, E. and Drogemeier, K.: The sensitivity of numerically simulated cyclic mesocyclogenesis to variations in model physical and computational parameters, Mon. Wea. Rev., 130, 26712691, 2002.

Buzzi, A. and Foschini, L.: Mesoscale meteorological features associated with heavy precipitation in the southern Alpine region, Meteorol. Atmos. Phys., 72, 131-146, 2000.

Buzzi, A., D'Isidoro, M., and Davolio, S.: A case study of an orographic cyclone south of the Alps during the MAP SOP, Quart J. Roy. Meteor. Soc., 129, 1795-1818, 2003 a. 
Buzzi, A., D'Isidoro, M., Davolio, S., Malguzzi, P.: Numerical assessment of MAP episodes of heavy precipitation using high resolution reanalyses and assimilation of surface data, Proceedings of the International Conference on Alpine Meteorology and MAP-Meeting 2003 (Brig, May 2003), Publications of MeteoSwiss, 66, 24-27, 2003b.

Bolla, R., Boni, G., Barbera, P. L., Lanza, L., Marchese, M., and Zappatore, S.: The tracking and prediction of high intensity rainstorms, Remote Sensing Reviews, 14, 151-184, 1996.

Deidda R.: Rainfall downscaling in a space-time multifractal framework, Water Resour. Res., 36, 1779-1794, 2000.

Doms, G. and Schaettler, U.: The nonhydrostatic limited-area model LM (Lokal-Modell) of DWD, Part I: Scientific documentation, Deutscher Wetterdienst (DWD), 1999.

Drofa, O. V.: The parameterization of microphysical processes for atmospheric numerical models, Il Nuovo Cimento, 26 C, $233-$ 262, 2003.

Ferraris, L., Rudari R., and Siccardi, F.: The uncertainty in the prediction of flash floods in the northern Mediterranean environment, J. Hydrometeorol., 3, 714-727, 2002.

Giannoni, F., Roth, G., and Rudari, R.: A Semi - Distributed Rainfall - Runoff Model Based on a Geomorphologic Approach, Phys. Chem. Earth, 25, 665-671, 2000.

Giannoni, F., Roth G., and Rudari, R.: A procedure for drainage network identification from geomorphology and its application to the prediction of the hydrologic response, Adv. Water Resour., 28, 6, 567-581, 2005.

Jacobson, I. and Heise, E.: A new economic method for the computation of the surface temperature in numerical models, Beitr. Phys. Atm., 55, 128-141, 1982.

Kain, J. S. and Fritsch, J. M.: A one-dimensional entraining/detraining plume model and its application in convective parameterization, J. Atmos. Sci. 47, 2784-2802, 1990.

Lanza, L. and Conti, M., Cloud tracking using satellite data for predicting the probability of heavy rainfall events in the Mediterranean area, Surveys in Geophys., 16, 163-181, 1995.

Louis J.F.: A parametric model of vertical eddy fluxes in the atmosphere, Boundary Layer Meteorology, 17, 187-2002, 1979.

Molteni, F., Buizza, R., Marsigli, C., Montani, A., Nerozzi, F., and Paccagnella, T.: A strategy for high-resolution ensemble prediction, Part I: definition of representative members and globalmodel experiments, Quart. J. Roy. Meteor. Soc., 127, 20692094, 2001.
Montani, A., Marsigli, C., Nerozzi, F., Paccagnella, T., and Buizza, R.: Performance of the ARPA-SMR limited-area ensemble prediction system: two flood cases, Nonlin. Processes Geophys., 8, 387-399, 2001,

SRef-ID: 1607-7946/npg/2001-8-387.

Montani, A., Marsigli, C., Nerozzi, F., Paccagnella, T., and Buizza, R.: The Soverato flood in Southern Italy: performance of global and limited-area ensemble forecasts, Nonlin. Processes Geophys., 10, 261-274, 2003,

SRef-ID: 1607-7946/npg/2003-10-261.

Morcrette, J. J.: Radiation and cloud radiative properties in the ECMWF operational weather forecast model, J. Geophys. Res., 96D, 9121-9132, 1991.

Morcrette, J. J., Clough, S. A., Mlawer, E. J., and Iacono, M. J.: Impact of a validated radiative transfer scheme, RRTM, on the ECMWF model climate and 10-day forecasts, ECMWF Technical Memo No. 252, 47 pp., 1998.

Mueller, C. K., Wilson, J. W., and Crook, N. A.: The Utility of Sounding and Mesonet Data to Nowcast Thunderstorm Initiation, Weather and Forecasting, 8, 132-146, 1993.

Rebora, N., Ferraris, L., von Hardenberg, J., and Provenzale. A.: Stocastic downscaling of LAM predictions: an example in the mediterranean area, Adv. Geosci., 3, 181-185, 2005.

Ritter, B. and Geleyn, J. F.: A comprehensive radiation scheme for numerical weather prediction models with potential applications in climate simulations, Mon. Wea. Rev., 120, 303-325, 1992.

Schultz, P.: An explicit cloud physics parameterization for operational numerical weather prediction, Mon. Wea. Rev. 123, 33313343, 1995.

SCS-CN: National Engineering handbook "Section 4: Hydrology" Soil Conservation Service USDA Washington DC, 1985.

Siccardi, F.: Rainstorm hazards and related disasters in the western Mediterranean region, Remote Sens. Rev., 14, 5-21, 1996.

Siccardi, F., Boni, G., Ferraris, L., and Rudari, R., A hydrometeorological approach for probabilistic flood forecast, J. Geophys. Res., 110, doi:10.1029/2004JD005314, 2005.

Tiedtke, M.: A comprehensive mass flux scheme for cumulus parameterization in large scale models., Mon. Wea. Rev., 117, 1779-1800, 1989.

Velden C. S. and Young, J. A.: Satellite Observations during TOGA COARE: Large-Scale Descriptive Overview, Mon. Wea. REv., 122, 2426-2441, 1994 